\title{
Adaptação transcultural dos Bancos de Itens de Ansiedade e Depressão do Patient-Reported Outcomes Measurement Information System (PROMIS) para língua portuguesa
}

\author{
Portuguese-language cultural adaptation of \\ the Items Banks of Anxiety and Depression of \\ the Patient-Reported Outcomes Measurement \\ Information System (PROMIS)
}

\author{
Traducción y adaptación cultural de Bancos de \\ Datos de Ansiedad y Depresión del Patient- \\ Reported Outcomes Measurement Information \\ System (PROMIS) en portugués
}

Natália Fontes Caputo de Castro 1
Carlos Henrique Alves de Rezende 1
Tânia Maria da Silva Mendonça ${ }^{1}$
Carlos Henrique Martins da Silva 1
Rogério de Melo Costa Pinto ${ }^{1}$

\section{Resumo}

$O$ Patient-Reported Outcome Measurement Information System (PROMIS), estruturado em domínios físicos e psicossociais, superou lacunas ao propor nova ferramenta de avaliação de resultados aplicáveis às doenças crônicas com base em técnicas avançadas de estatística (TRI) e testes adaptativos computadorizados (CAT). O objetivo do estudo foi adaptar culturalmente os Bancos de Itens de Ansiedade e Depressão do PROMIS para a língua portuguesa. O processo seguiu rigorosas recomendações do FACIT por meio da tradução avançada, reconciliação, retrotradução, revisão do FACIT, revisores independentes, finalização das etapas pelo FACIT, pré-teste e incorporação dos resultados do préteste. A versão traduzida foi pré-testada em dez pacientes, sendo necessária a modificação nos itens 3, 46 e 53 de Ansiedade e no item 46 de Depressão. As alterações alcançaram a equivalência de significado e a versão final foi compatível com as habilidades linguísticas e culturais da população brasileira. Concluiu-se que a versão traduzida é semântica e conceitualmente equivalente aos originais.

Translating; Anxiety; Depression; Quality of Life
Tradução; Ansiedade; Depressão; Qualidade de Vida 


\section{Introdução}

A percepção do paciente em relação ao seu estado de saúde permitiu a inclusão de outros parâmetros para a avaliação da saúde, especificamente questionários que mensuram o impacto das doenças crônicas sobre a vida das pessoas 1 .

Com o intuito de superar algumas limitações dos questionários atuais, pesquisadores norte-americanos desenvolveram o PatientReported Outcomes Measurement Infomation System (PROMIS) por meio de modernas técnicas de estatísticas, como a Teoria de Resposta ao Item (TRI) e do avanço da tecnologia da informação, como o Teste Adaptativo Computadorizado (CAT). O sistema PROMIS permite uma avaliação mais consistente da qualidade de vida relacionada à saúde, além de minimizar o número de itens administrados por disponiblizadar apenas itens correspondentes ao nível de comprometimento do paciente $2,3,4,5,6$.

Os Bancos de Itens de Ansiedade e Depressão, considerados fatores de risco para doenças crônicas, constituem o domínio Dificuldades Emocionais do PROMIS (versão 1.0). O Banco de Depressão é composto por indicadores cognitivos, afetivos e comportamentais, e o de Ansiedade, por indicadores somáticos 7 .

A versão original dos Bancos de Itens de Ansiedade e Depressão do PROMIS está disponível no inglês americano e para a sua utilização em outros idiomas é necessária a tradução e adaptação cultural para posterior validação ${ }^{8}$. Os desenvolvedores do PROMIS preconizam que esse processo contemple uma tradução universal para produção de uma versão comum para países que falam o mesmo idioma 9,10.

O objetivo do estudo foi realizar a tradução e adaptação cultural dos Bancos de Itens de Ansiedade e Depressão do domínio Dificuldades Emocionais do PROMIS (versão 1.0) para a língua portuguesa.

\section{Metodologia}

Este é um estudo para adaptação transcultural e linguística dos Bancos de Itens de Ansiedade e Depressão do PROMIS, realizado após aprovação do Comitê de Ética em Pesquisa da Universidade Federal de Uberlândia e permissão concedida pelo PROMIS. O processo foi conduzido segundo as recomendações publicadas pelo Funcional Assessment of Chronic Ilness Therapy (FACIT) 10 e constituído por oito etapas conforme Figura 1.

Os itens dos Bancos de Ansiedade e Depressão foram traduzidos de forma independente $\mathrm{e}$ simultânea por dois tradutores. Em seguida, as versões foram reconciliadas e, quando necessário, uma tradução alternativa foi proposta a fim de garantir a compatibilidade linguística. A versão reconciliada foi retrotraduzida para o inglês americano a fim de garantir a equivalência de significado com o item original. Os coordenadores do PROMIS analisaram cada item retrotraduzido para identificar as traduções inapropriadas e ambíguas. Posteriormente, análises e comentários foram encaminhados aos revisores independentes a fim de selecionar a melhor tradução para cada item. Para contemplar uma versão universal, revisores do Brasil e de Portugal participaram do processo com o intuito de harmonizar as diferenças linguísticas existentes nos dois países. As versões originadas foram enviadas aos coordenadores do PROMIS para produção da versão pré-teste dos bancos de itens para serem pré-testadas em dez pacientes, acompanhados no Ambulatório Central do Hospital de Clínicas da Universidade Federal de Uberlândia (HC-UFU).

Os participantes assinaram o Termo de Consentimento Livre e Esclarecido, responderam a um questionário sociodemográfico, à versão préteste e às entrevistas de esclarecimento: retrospectiva e cognitiva. Foram consideradas respostas satisfatórias aquelas com porcentagem igual ou maior que $80 \%$.

\section{Resultados}

Identificou-se prejuízo na equivalência semântica nos itens 1, 2 e 12 do Banco de Ansiedade e nos itens 6 e 28 do Banco de Depressão (Tabela 1).

As retrotraduções dos itens 1, 2 e 12 do Banco de Ansiedade e do item 6 do Banco de Depressão não refletiram adequadamente o conceito da versão original. No Banco de Itens de Ansiedade, a expressão fearful do item 1 foi retrotraduzida para afraid; no item 2, frightned para scared; no 12, upset para disgusted e no 6, helpless para abandoned. Além disso, foi necessário um consenso para a tradução da palavra overwhelming, que recebeu diferentes traduções: "devastadoras”, "excessivas”, “opressivas”, “catastróficas” e "esmagadoras". E o verbo felt foi retrotraduzido para believed e thought.

No item 3 do Banco de Ansiedade, o revisor independente modificou a frase para "Fiquei assustado(a) com meu nervosismo”. A expressão "atividades públicas" foi substituída para "atividades sociais", o que assegurou a equivalência cultural do item 24. No item 5 do Banco de Depressão, optou-se por uma linguagem coloquial que foi traduzida para "Eu senti que não tinha perspectiva para o futuro”. Jáno item 35 do mesmo 
Metodologia de tradução.

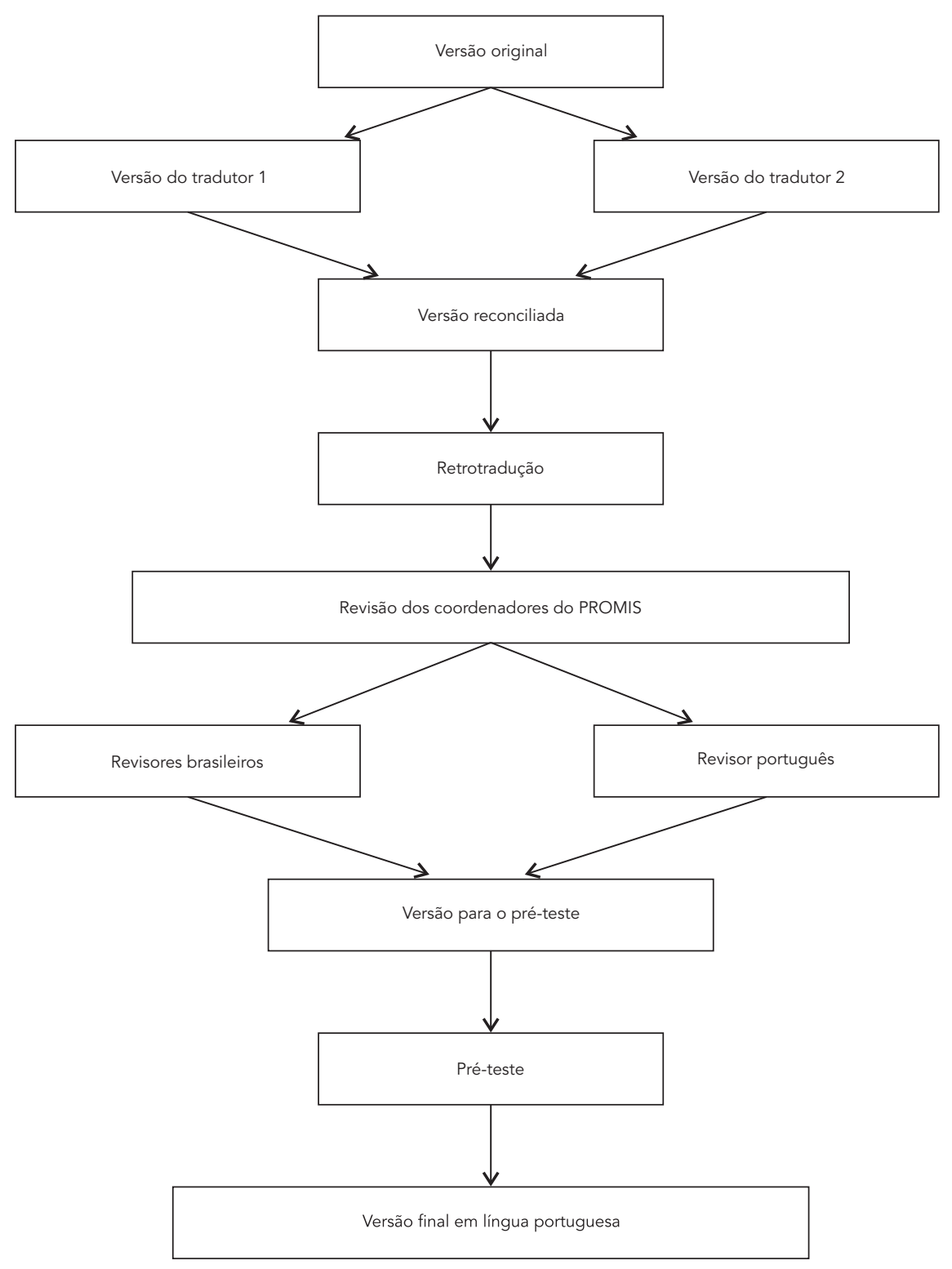

PROMIS: Patient-Reported Outcomes Measurement Information System.

banco, a equivalência semântica foi assegurada pelo revisor ao propor "Percebi que as coisas em minha vida estavam me sufocando" (Tabela 1).

O revisor brasileiro substituiu a palavra "trêmulo" por "tremores". Em outro item, o revisor de Portugal sugeriu que a palavra helpless fosse traduzida para "impotente", mas o revisor brasileiro optou pela expressão "incapaz".
Houve incompreensão nos itens 3, 46 e 53 do Banco de Ansiedade. Os itens 18 e 40 desse mesmo banco alcançaram a equivalência semântica com os itens originais, no entanto sofreram alguns ajustes (Tabela 1).

Houve incompreensão no item 46 do Banco de Depressão, por isto ele foi submetido à nova tradução. $\mathrm{O}$ item 4 alcançou a equivalência 
Tabela 1

Versões do pré-teste e final dos Bancos de Itens de Ansiedade e Depressão do Patient-Reported Outcomes Measurement Information System (PROMIS)

\begin{tabular}{|c|c|c|c|}
\hline Banco & Item & Versão pré-teste & Versão final \\
\hline \multirow[t]{6}{*}{ Ansiedade } & 3 & Fiquei assustado(a) com o meu nervosismo & Fiquei assustado(a) com a minha agitação \\
\hline & 18 & Tive sensações repentinas de pânico & De repente tive sensações de pânico \\
\hline & 40 & Achei difícil concentrar-me em algo que não fosse & Achei difícil concentrar-me em qualquer coisa a não ser \\
\hline & & a minha ansiedade & na minha ansiedade \\
\hline & 46 & Senti-me nervoso(a) & Senti-me agitado(a) \\
\hline & 53 & Senti-me aflito(a) & Senti-me angustiado(a) \\
\hline \multirow[t]{2}{*}{ Depressão } & 4 & Senti-me inútil & Senti-me sem valor e sem importância (inútil para as pessoas) \\
\hline & 46 & Senti-me pessimista & Senti-me pessimista (vi as coisas de forma negativa) \\
\hline
\end{tabular}

semântica esperada, porém optou-se pelo ajuste da versão final (Tabelas 1 e 2).

\section{Discussão}

O processo de adaptação cultural dos Bancos de Itens de Ansiedade e Depressão do PROMIS para a língua portugesa foi realizado por meio de rigorosos padrões metodológicos. A inclusão dos revisores independentes após a etapa de retrotradução e o uso das entrevistas de esclarecimento na etapa de pré-teste contribuíram para que a versão final dos dois bancos alcançasse um alto nível de qualidade para uso em países de língua portuguesa.

As traduções alternativas propostas pelo reconciliador possibilitaram maior objetividade ao processo e a utilização de uma linguagem coloquial. De modo geral, o método de retrotradução foi eficaz ao garantir a qualidade do processo de tradução. A comparação das versões retrotraduzidas com a versão original dos itens contribuiu de modo significativo para a avaliação da equivalência de significado por meio da recomendação de mudanças nas retrotraduções de though e believed, pois ambas não corresponderam adequadamente à proposta original, já que a expressão felt corresponde a "sentir". O termo thought pode conferir ênfase às habilidades cognitivas e "acreditar” pode refletir uma crença ou convicção que não necessariamente está relacionada à percepção do paciente.

Com o intuito de alcançar a equivalência semântica no item 3 do Banco de Ansiedade, o revisor optou pela reestruturação da frase, fornecendo um significado completo e compreensível ao item. A equivalência cultural do item 24, do mesmo banco, pôde ser obtida por meio da substituição da expressão "atividades públicas" por "atividades sociais", pois o primeiro termo não correspondeu ao vocabulário usualmente utilizado pela nossa população. Foram elaboradas novas versões para os itens 5 e 35 do Banco de Depressão, com a finalidade de melhorar a compressão e utilizar uma linguagem mais coloquial. O estabelecimento de uma versão universal demonstrou que algumas diferenças foram harmonizadas, como a mudança do termo "trêmulo" para "tremores", uma vez que em Portugal a palavra é acentuada de forma diferente "trémulos". O revisor de Portugal sugeriu que a expressão helpless fosse traduzida para "impotente", no entanto, este vocábulo é popularmente associado à dificuldade de ereção sexual, o que poderia causar constrangimento aos pacientes brasileiros. Dessa forma, a palavra "incapaz" mostrou-se mais adequada.

As entrevistas de esclarecimento têm sido apontadas por diversos estudos como um método significativo para a obtenção de feedback do respondente sobre os itens de um instrumento, uma vez que auxilia na opção de finalizar de maneira efetiva o processo de tradução e adaptação cultural.

O significado proposto no item original para a expressão nervous corresponde à "agitação psicomotora/inquietação" e não a sentimentos relacionados à "irritabilidade/nervosismo", como foi compreendido pela maioria dos respondentes. A palavra "aflito" ficou inadequada por parecer distante do coloquialismo da língua falada pela população brasileira. No Banco de Itens de Depressão, o termo pessimistic não correspondeu ao significado proposto, pois os pacientes apresentaram dificuldades de compreensão devido à pouca utilização desta expressão no cotidiano da população pré-testada. O ajuste dos itens 18 e 40 do Banco de Ansiedade e do item 4 do Banco de Depressão, que mesmo tendo atingido uma por- 
Tabela 2

Versão dos Bancos de Itens de Ansiedade e Depressão do Patient-Reported Outcomes Measurement Information System (PROMIS) na língua portuguesa.

\begin{tabular}{|c|c|c|c|}
\hline Item & Ansiedade & Item & Depressão \\
\hline 1 & Senti-me apreensivo(a) & 4 & Senti-me sem valor e sem importância (inútil para as pessoas) \\
\hline 2 & Senti-me amedrontado(a) & 5 & Senti que eu não tinha expectativas para o futuro \\
\hline 3 & Fiquei assustado(a) com a minha agitação & 6 & Senti-me incapaz \\
\hline 5 & Senti-me ansioso(a) & 7 & Afastei-me das pessoas \\
\hline 7 & Senti que precisava de ajuda para a minha ansiedade & 9 & Senti que nada podia animar-me \\
\hline 8 & Fiquei preocupado(a) com a minha saúde mental & 14 & Senti que eu não era bom/boa como as outras pessoas \\
\hline 12 & Senti-me chateado(a) & 17 & Senti-me triste \\
\hline 13 & O meu coração bateu forte ou acelerado & 19 & Senti que eu queria desistir de tudo \\
\hline 16 & Fiquei ansioso(a) quando a minha rotina normal foi alterada & 21 & Senti que eu era o(a) culpado(a) pelas coisas \\
\hline 18 & De repente tive sensações de pânico & 22 & Senti-me um fracassado(a) \\
\hline 20 & Fiquei assustado(a) com facilidade & 23 & Tive dificuldade em sentir-me próximo(a) das pessoas \\
\hline 21 & Tive dificuldade em prestar atenção & 26 & Senti-me desapontado(a) comigo mesmo(a) \\
\hline 24 & Evitei atividades sociais ou lugares públicos & 27 & Senti que eu não fazia falta \\
\hline 26 & Senti-me inquieto(a) & 28 & Senti-me sozinho(a) \\
\hline 27 & Senti que algo terrível poderia acontecer & 28 & Senti-me deprimido(a) \\
\hline 30 & Senti-me preocupado(a) & 30 & Tive dificuldade em tomar decisões \\
\hline 33 & Senti-me aterrorizado(a) & 31 & Senti-me desencorajado(a) em relação ao futuro \\
\hline 37 & Fiquei preocupado(a) com a reação dos outros em relação a mim & 35 & Achei que as coisas na minha vida estavam sufocando-me \\
\hline 40 & $\begin{array}{l}\text { Achei difícil concentrar-me em qualquer coisa a não ser } \\
\qquad \text { na minha ansiedade }\end{array}$ & 36 & Senti-me infeliz \\
\hline 41 & As minhas preocupações dominaram-me completamente & 39 & Senti que não tinha razão para viver \\
\hline 44 & Tive contrações involuntárias ou tremores musculares & 41 & Senti-me sem esperança \\
\hline 46 & Senti-me agitado(a) & 42 & Senti-me ignorado(a) pelas pessoas \\
\hline 47 & Senti-me indeciso(a) & 44 & Senti-me chateado(a) sem motivo \\
\hline 48 & Muitas situações deixaram-me preocupado(a) & 45 & Senti que nada era interessante \\
\hline 49 & Tive dificuldade em dormir & 46 & Senti-me pessimista (vi as coisas de forma negativa) \\
\hline 51 & Tive dificuldade em relaxar & 48 & Senti que a minha vida estava vazia \\
\hline 53 & Senti-me angustiado(a) & 50 & Senti-me culpado(a) \\
\hline 54 & Senti-me tenso(a) & 54 & Senti-me emocionalmente esgotado(a) \\
\hline 55 & Tive dificuldade em me acalmar & & \\
\hline
\end{tabular}

centagem satisfatória de compreensão, ocorreu a fim de melhorar o entendimento.

Concluindo, a versão traduzida dos Bancos de Itens de Ansiedade e Depressão do PROMIS apresenta equivalência em relação aos originais para a população brasileira. Os itens adaptados para a população brasileira serão disponibilizados assim que o processo de validação seja concluído. 


\section{Resumen}

El Patient-Reported Outcomes Measurement Information System (PROMIS), estructurado en dominio fisico y psicosocial, superó deficiencias proponiendo una nueva herramienta para la evaluación de resultados aplicables a enfermedades crónicas, usando técnicas avanzadas de estadisticas (TRI) y pruebas de adaptación computarizada (CAT). El objetivo fue adaptar culturalmente items de los Bancos Ansiedad y Depresión, PROMIS en portugués. El proceso siguió estrictas recomendaciones del FACIT, a través de traducción avanzada, reconciliación, traducción inversa, revisión del FACIT, revisores independientes, pre-prueba e incorporación de los resultados de pre-test. La versión traducida fue pre-prueba en diez pacientes, y los artículos 3, 46 y 53 (ansiedad) y el 46 (depresión) se cambiaron. Los cambios llegaron a la equivalencia de significado y la nueva versión fue compatible con las competencias lingüísticas y culturales de la población brasileña. Se concluyó que la versión traducida es semántica y conceptualmente equivalente a la original.

Traducción; Ansiedad; Depresión; Calidad de Vida

\section{Colaboradores}

N. F. C. Castro, C. H. A. Rezende, T. M. S. Mendonça, C. H. M. Silva e R. M. C. Pinto contribuíram no projeto e delineamento, no levantamento, análise e interpretação dos dados, na elaboração do manuscrito e na aprovação da versão a ser publicada.

\section{Agradecimentos}

Os autores agradecem à FAPEMIG pelo apoio financeiro e ao Grupo de Pesquisa de Qualidade de Vida da Faculdade de Medicina da Universidade Federal de Uberlândia (UFU). Aos coordenadores do processo de tradução, Helena Correa e Benjamin Arnold, representando os desenvolvedores do PROMIS.

\section{Referências}

1. Patrick DL, Chiang YP. Measurement of health outcomes in treatment effectiveness evaluations: conceptual and methodological challenges. Med Care 2000; 38(9 Suppl):14-25.

2. Chakravaty FE, Bjorner BJ, Fries FJ. Improving patient reported outcomes using item response theory and computerized adaptive testing. J Rheumatol 2007; 34:1426-31.

3. Fries FJ, Bruce B, Cella D. The promise of PROMIS: using item response theory to improve assessment of patient-reported outcomes. Clin Exp Rheumatol 2005; 39:53-7.

4. Cella D, Riley W, Stone A, Rothrock N, Reeve B, Yount S, et al. Initial adult health item banks and first wave testing of the Patient-Reported Outcomes Measurement Outcomes Information System (PROMISTM) Network: 2005-2008. J Clin Epidemiol 2010; 63:1179-94.

5. Cella D, Yount S, Rothrock N, Gershon R, Cook K, Reeve B, et al. The Patient-Reported Outcomes Measurement Information System (PROMIS): progress of an NIH Roadmap cooperative group during its first two years. Med Care 2007; 45:3-11.

6. PROMIS Cooperative Group. Manual for the Patient-Reported Outcomes Measurement Information System (PROMIS®) Version 1.1. http://www. nihpromis.org (acessado em 10/Mai/2011).

7. Pilkonis PA, Choi WS, Reise PS, Stover MA, Cella D. Item banks for measuring emotional distress from the Patient-Reported Outcomes Measurement Information System (PROMIS): depression, anxiety, and anger. Assessment 2011; 12:263-83.

8. Guillemin F, Bombardier C, Beaton D. Cross-cultural adaptation of health-related quality of life measures: literature review and proposed guidelines. J Clin Epidemiol 1993; 46:1417-32.

9. Bonomi AE, Cella DF, Hahn EA, Bjordal K, Sperner-Unterweger B, Gangeri L, et al. Multilingual translation of the Functional Assessment of Cancer Therapy (FACT) quality of life measurement system. Qual Life Res 1996; 5:309-20.

10. Eremenco SL, Cella D, Arnold BJ. A comprehensive method for the translation and cross-cultural validation of health status questionnaires. Eval Health Prof 2005; 28:212-32.

Recebido em 07/Mar/2013

Versão final reapresentada em 19/Dez/2013

Aprovado em 06/Jan/2014 